\title{
DEVICE FOR MEASURING AND MAINTAINING THE WATER'S LEVEL IN A TANK
}

\author{
Ionuț Craiu ${ }^{1}$, Nicolae Fidel ${ }^{2}$, Mihail-Florin $\mathrm{Stan}^{3}$, Ramona Chirescu ${ }^{4}$, Florian Ion $^{5}$, \\ Valahia University of Targoviste, Faculty of Electrical Engineering ${ }^{1,2,3,4,5}$, \\ ionutcraiu10@yahoo.ro1, nicolae.fidel@valahia.ro², flo.stan@gmail.com3, corvinchirescu@yahoo.ro ${ }^{4}$, \\ ionflorian@yahoo.com
}

\begin{abstract}
This paper presents a device for measuring and maintaining the constant water level in a tank. The device uses a capacity sensor to measure the water level. The sensor has a DC voltage output proportional to the water level in the tank. This voltage can be used in other automation too. The water level, in percentages, is displayed on a vu meter with 8 LEDs. The circuit for maintaining the constant level consists in a comparator with hysteresis. The level of the water is adjustable, using a reference voltage from a potentiometer (marked in percentages). The level of the water is compared with the prescribed level and the difference between them commands the start/stop of the water pump which supplies the tank through a relay. The device is powered by $220 \mathrm{~V}$ and is isolated from the environment.
\end{abstract}

Keywords: capacitive level sensor, water level monitoring, maintaining constant water level.

\section{INTRODUCTION}

The work presented below was based on the practical need for maintaining the constant water level in a tank.

Such an application is found at the URL [1] address. The simplicity of the scheme is easily noticed - using the CD4093 logic gates with Schmitt trigger to control a transistor with a transistor having a relay on collector. The relay turns on/off the motor pump of the tank. The disadvantage of the system is the fact that the water level cannot be adjusted. The level is given by the position of two sensors A and B - B; A for the maximum level and $\mathrm{B}$ for the minimum level. The scheme does not give any information about intermediate level of water between the minimum and maximum.

At the web address [2] there is another application. Simplicity is noticed as in the previous one - using transistors and multiple relay contacts. The disadvantage is the use of fixed points to close/open the relay contacts. The resistance of water is what controls the base currents of the transistors; hence, the second drawback of the circuit is the variable resistance of water. The intermediate level of water is not monitored.

Another scheme is proposed on the website [3]. The circuit uses an RS flip-flop with NOR gates controlled by the water level in the tank. Like the previous scheme, the water level is fixed and is used to control the switching of the flip-flop.

The presentation of other applications [4], [5] can continue, but I decided to stop here in order to give a detailed description of the automation that the paper presents.

\section{THE DEVICE DESIGN}

The premises of automation are:

- The dimensions of the tank must not be an element of coercion or, in other words, the automation can be used with any type of tank.

- The tank must be intact - not to be ruptured to mount the sensors.

- The resistance of water must not be used as the control element.

- The water level must be adjustable from the outside of tank - on the front panel of the circuit.

- The water pump of the tank must be isolated from the control circuit.

Based on the above considerations, we proposed automation with the following parameters:

- The circuit should be simple, to be achieved with transistors and operational amplifiers,

- To be low cost,

- The water level should be sensed with a capacitive sensor (isolated from the environment)

- The water level in the tank should be adjustable,

- The water level should be displayed on a Bar Graph,

- The motor of the water supply tank should be controlled by a relay.

The method of measurement in the frequency domain was chosen to measure the water level.

The equation for calculating the capacity of a capacitor is:

$$
C=\varepsilon_{r} \varepsilon_{0} \frac{S}{d}=K \times S
$$

$\mathrm{Er}$ - relative permittivity of water (constant in our case),

Eo - electric permittivity of vacuum equal to $8,854187817 \times 10^{-12} \mathrm{~F} / \mathrm{m}$,

$\mathrm{S}$ - the surface of the capacitor plates,

$\mathrm{d}$ - the distance between the plates (constant).

Consequently, the value of the capacity will be given by the multiplication of $\mathrm{K}$ - the constant on the (1) formula, 
and $\mathrm{S}$ - the area of the capacitor plates. The variation of $\mathrm{S}$ is proportional with the water level of the tank.

The water level sensor is constituted under the block diagram in Figure 1.

The physical realization of the capacitive sensor consists of two parallel conductors (or two tracks of copper on a PCB) that had their minimum capacity measured (in the air), and were totally immersed in water - having their maximum capacity measured.

It was found that the maximum values are dependent on the shape, size and the placement of the plates.

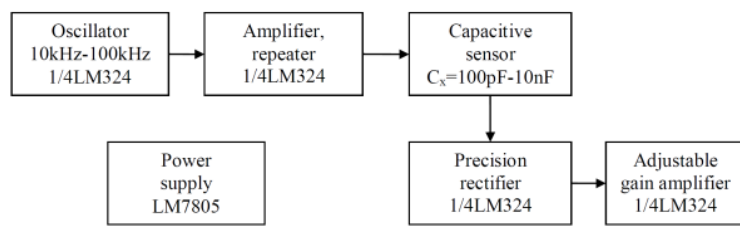

Figure 1. The block diagram of the capacitive sensor.

\section{SIMULATION OF OPERATION}

Figure 2 presents three variants - four insulated copper tracks - VAR1, two parallel copper wires - VAR2, two twisted cables - VAR3.

The adjustable frequency oscillator is made with one operational amplifier from the LM324 [6].

Because the oscillation should not be influenced or depreciated by the capacity of the capacitive sensor, we used a repeater made with another amplifier of the same LM324.

The rectangular signal from the repeater, passed through the sensor, is brought to the terminals of a double precision rectifier done with an operational amplifier from the LM324 circuit [7].

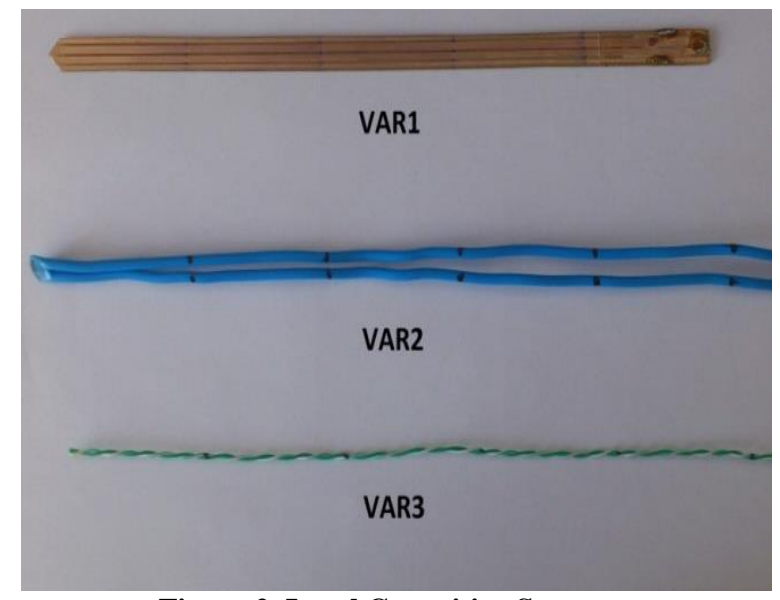

Figure 2. Level Capacitive Sensors.

The waveform of the signal at the output of the precision rectifier is shown in Figure 3. The program used for simulations was Multisim 12.0 of the National Instruments.

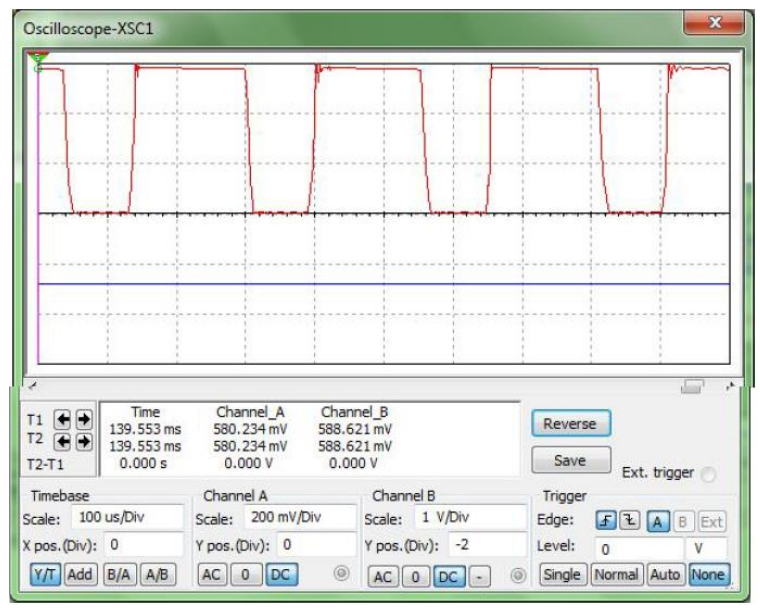

Figure 3. The signal at the output of the precision rectifier.

The last operational amplifier of LM324 is used as a non-inverting adjustable gain amplifier to determine the value of signal given by the precision rectifier.

The value of the amplification is given by (2):

$$
V o=V_{C 6}=\left(1+\frac{R_{45}}{R_{46}}\right) \times V_{C 5},
$$

The equation is equal to $1.68 \mathrm{~V}$ for $\mathrm{R} 46=6.8 \mathrm{k} \Omega$ and $\mathrm{R} 45=10 \mathrm{k} \Omega$.

Table nr 1 presents some results obtained by simulation for different values of the capacitive sensor at the frequencies of $28.2 \mathrm{kHz}$ and $4.0 \mathrm{kHz}$.

Table 1. The output voltage with different values of the capacitive sensor.

\begin{tabular}{|c|c|c|}
\hline fosc [kHz] & Csensor [pF] & Vout [V] \\
\hline \multirow{3}{*}{28.2} & 5 & 1.68 \\
\cline { 2 - 3 } & 10 & 2.42 \\
\cline { 2 - 3 } & 20 & 2.82 \\
\cline { 2 - 3 } & 40 & 3.9 \\
\cline { 2 - 3 } & 80 & 3.1 \\
\hline \multirow{4}{*}{4,5} & 140 & 3.14 \\
\cline { 2 - 3 } & 1400 & 3.5 \\
\cline { 2 - 3 } & 14000 & 3.65 \\
\cline { 2 - 3 } & 140000 & 3.75 \\
\hline
\end{tabular}


In Figure 4 the output voltage is $176 \mathrm{mV}$ at a frequency of oscillation of $4.0 \mathrm{kHz}$ and the capacity of the sensor of $1 \mathrm{nF}$.

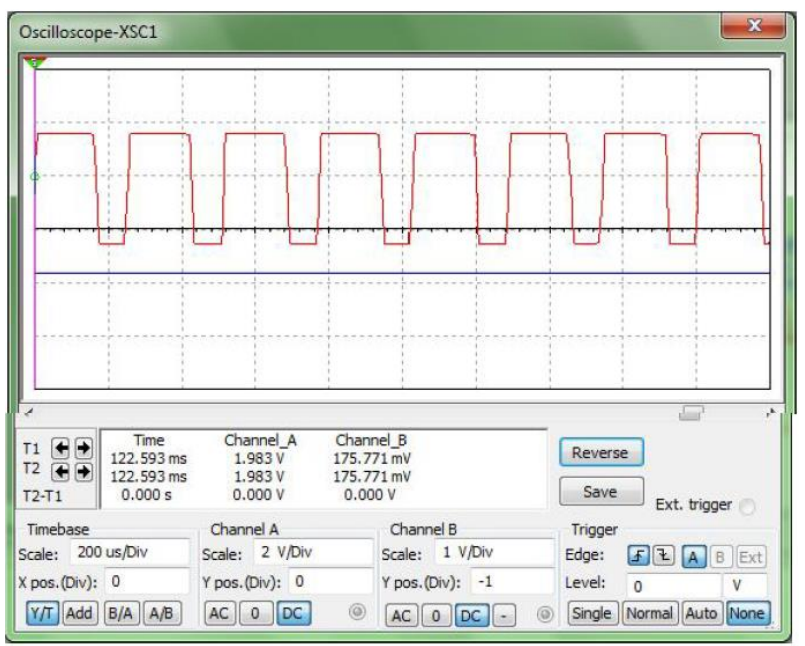

Figure 4. The output voltage at the frequency of $4.0 \mathrm{kHz}$ and Csensor of $1 \mathrm{nF}$.

\section{THE DEVICE PROTYPE}

In various fields of application - residential houses supplies, industrial units, hospitals, etc., the water level must be measured and kept constant for a certain time. It sets an interval in which the water level must not fall below a certain value - the minimum value, or must not exceed a certain value - the maximum value. These limits must be controlled by the user.

The functional blocks of the automation system is presented in Figure 5:

- The circuit that measures the water level; the output voltage is directly proportional with the Vlevel,

- A Bar Graph display type with 8 LED - to display the current level of the water in the tank,

- An inverting amplifier with a hysteresis of $0.1 \mathrm{~V}$ corresponding to a variation of level of $2.5 \%$ of the maximum value, with adjustment possibility,

- A relay to switch on/off the water pump,

- A power supply.

\subsection{The control circuit of water pump}

To control the water level in the tank a water pump driven by a relay should be used. Starting and stopping the pump should be at a certain predetermined value of the water level in the tank.

The circuit is designed with hysteresis and an inverting amplifier with a pump' control relay.

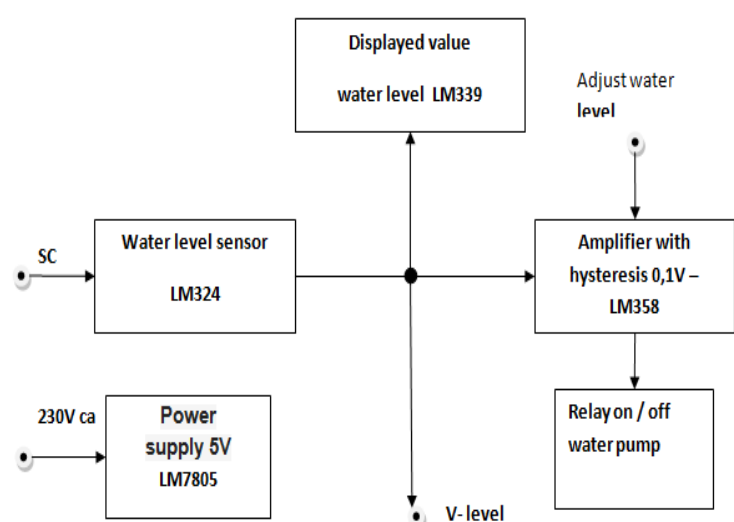

Figure 5. The block diagram of measuring and keeping constant the water level of a tank.

The value of the level that stops the pump is adjusted with a potentiometer.

When the output operational amplifier is at $3.5 \mathrm{~V}$ (corresponding to a level lower than the adjusted level), through a transistor, a relay is energized and its contacts activate the pump. The pump status is indicated by a LED.

If the water level exceeds the set value, the output voltage of amplifier is $0.1 \mathrm{~V}$; the transistor is blocked, the contacts of relay are open and the pump stops.

The level of water is displayed by 2 integrated circuits LM 339, which contain four comparators, that each controls 4 LEDs. This display indicates different levels of the water for $10,20,40,50,60,70,80$ and $90 \%$ of the maximum level.

\section{EXPERIMENTAL RESULTS}

To simulate the system of maintaining the water level in a tank at a certain value, we designed a prototype circuit on a PCB of $160 \times 100 \mathrm{~mm}$.

The circuit is powered from 230Vac. With a buck transformer, a bridge rectifier and a capacitor are obtained by filtering $8.2 \mathrm{Vdc}$ which ensure the proper functioning of the linear regulator LM7805. The capacitive sensor is connected to the input SC in Figure 5 .

The capacitive sensor is done in three variants of 100 , 300 and $1000 \mathrm{~mm}$ length to determine the water levels in tanks of different heights. The first version - hereinafter referred to as VAR1 in Figure 2 is made of $12 \mathrm{~mm}$ width of glass-reinforced epoxy laminate (FR4), plain coated, 4 tracks over the entire length of $300 \mathrm{~mm}$. Its capacity after the measurement is between 12 and $36 \mathrm{pF}$ in the air (adding the capacity of the cable connection).

Other variants tested:

- Two wires of $2 \mathrm{~mm}$ diameter of copper insulated, glued parallel to each other (the distance between the two wires of insulation thickness about $3 \mathrm{~mm}$ ). 
- Two twisted wires $1000 \mathrm{~mm}$ length of Cat5e Ethernet cable.

With all 3 variants the device works well. The user only has to change the position of the potentiometer for the desired water level of the tank. The voltage proportional to the water level can be read at the Vnivel terminal of device.

If it is desired to use this value in automation where other parameters should also be supervised - like: temperature, different concentrations, etc.), the terminal Vnivel should be connected to an analog input of a PLC.

The closing/opening of relay contacts controls the start/stop of the pump used to fill the tank.

The water level in the tank is displayed on 8 LEDs connected to the outputs of two comparator circuits LM339 [8].

\subsection{Measurements performed on the prototype}

The first signal viewed on the oscilloscope channel CH1 is the output voltage of the oscillator. The signal displayed on $\mathrm{CH} 2$ channel is the voltage of output Vnivel. The measurement is performed with the capacitive sensor VAR1 in free air (Csenzor = $12 \mathrm{pF})$. The voltage at the output Vnivel is $0.487 \mathrm{~V}$ Figure 6 [9].

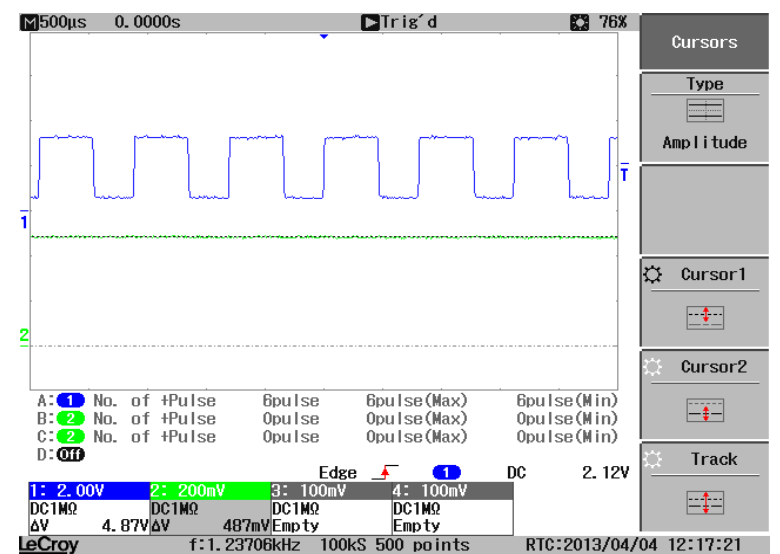

Figure 6. The output Vnivel with Csenzor $=12 \mathrm{pF}$.

In Figure 7 [9] the voltage on sensor is shown, with the sensor VAR1 placed in a bowl with $130 \mathrm{~mm}$ height of water. The output voltage Vnivel is $1.24 \mathrm{~V}$ in these circumstances. The channel $\mathrm{CH} 2$ displays the output of the oscillator and the signal of channel $\mathrm{CH} 2$ is the voltage on the sensor.

The waveforms viewed with different values of the capacitive sensor at different water levels are very close to those shown in the Table 2; only the output voltage Vnivel is changed. The maximum of $3.41 \mathrm{~V}$ is achieved when the capacitive sensor is immersed in the tank 250mm - Figure 8 [9].

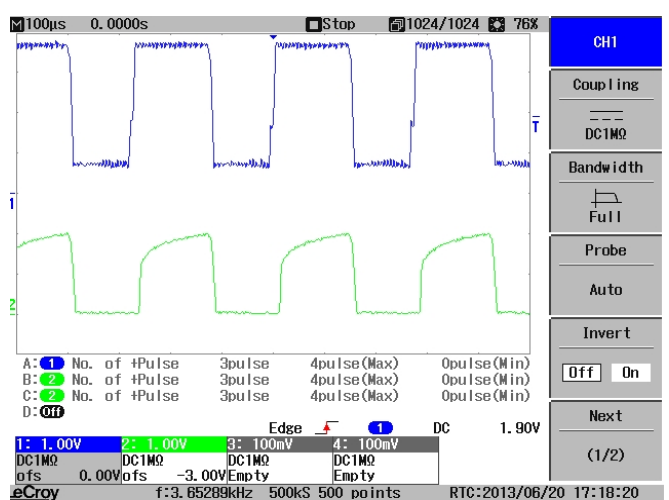

Figure 7. The voltage on the sensor of the device with the VAR1 sensor, immersed in $130 \mathrm{~mm}$ water.

In Table 2 are shown the measurements obtained with the three types of sensors - VAR1, VAR2 and VAR3 at different heights in the tank water.

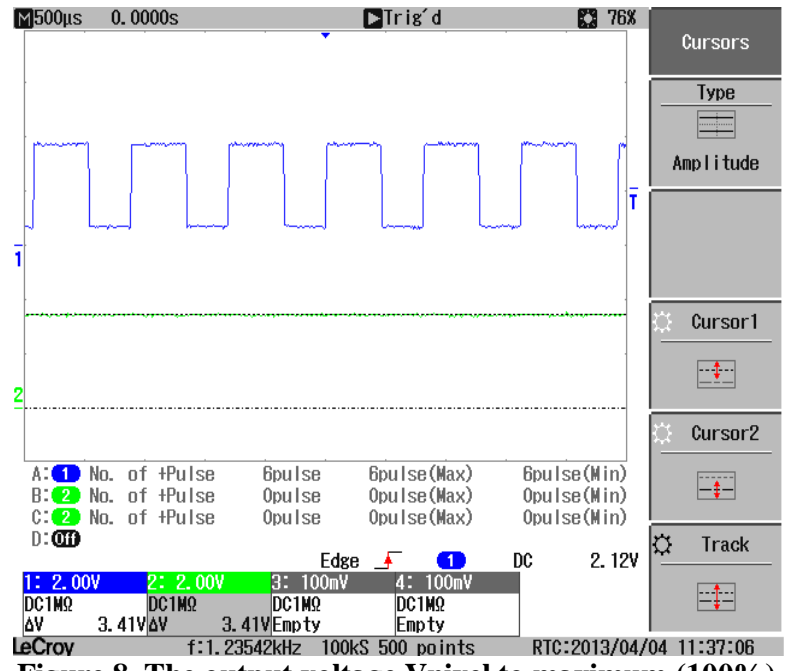

Figure 8. The output voltage Vnivel to maximum $(100 \%)$ immersed in $250 \mathrm{~mm}$ of water.

Table 2. The output voltage Vnivel at different water heights with three types of sensors.

\begin{tabular}{|c|c|c|}
\hline Sensor version & $\begin{array}{c}\text { Water height } \\
{[\mathbf{m m}]}\end{array}$ & $\begin{array}{c}\text { Vnivel } \\
{[\mathbf{V}]}\end{array}$ \\
\hline \multirow{5}{*}{ VAR1 } & 0 & 0.26 \\
\cline { 2 - 3 } & 20 & 0.36 \\
\cline { 2 - 3 } & 30 & 0.44 \\
\cline { 2 - 3 } & 40 & 0.47 \\
\cline { 2 - 3 } & 50 & 0.50 \\
\cline { 2 - 3 } & 80 & 0.90 \\
\cline { 2 - 3 } & 100 & 1.30 \\
\hline \multirow{5}{*}{ VAR2 } & 125 & 1.40 \\
\cline { 2 - 3 } & 0 & 0.60 \\
\cline { 2 - 3 } & 50 & 0.67 \\
\cline { 2 - 3 } & 100 & 0.77 \\
\cline { 2 - 3 } & 150 & 0.88 \\
\hline \multirow{5}{*}{ VAR3 } & 200 & 1.00 \\
\cline { 2 - 3 } & 250 & 1.11 \\
\hline & 0 & 0.58 \\
\hline
\end{tabular}




\begin{tabular}{|l|l|l|}
\hline \multirow{4}{*}{} & 100 & 0.74 \\
\cline { 2 - 3 } & 150 & 0.80 \\
\cline { 2 - 3 } & 200 & 0.87 \\
\cline { 2 - 3 } & 250 & 0.93 \\
\hline
\end{tabular}

In Figure 9 [9] the graph of variation of output voltage Vnivel with sensor VAR3 is shown. Here, may be seen the good linearity of the output voltage to the height of the water column in the tank.

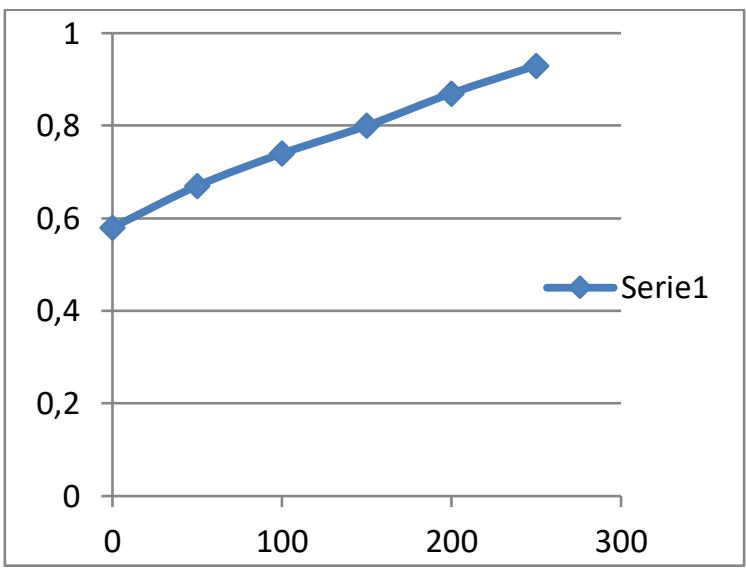

Figure 9. The graph of output voltage to the height of water in the tank with the VAR3 sensor.

Figure 10 presents a picture of the device prototype. All LEDs are on - the tank is full of water, and the LED that shows the status of the motor pump is off.

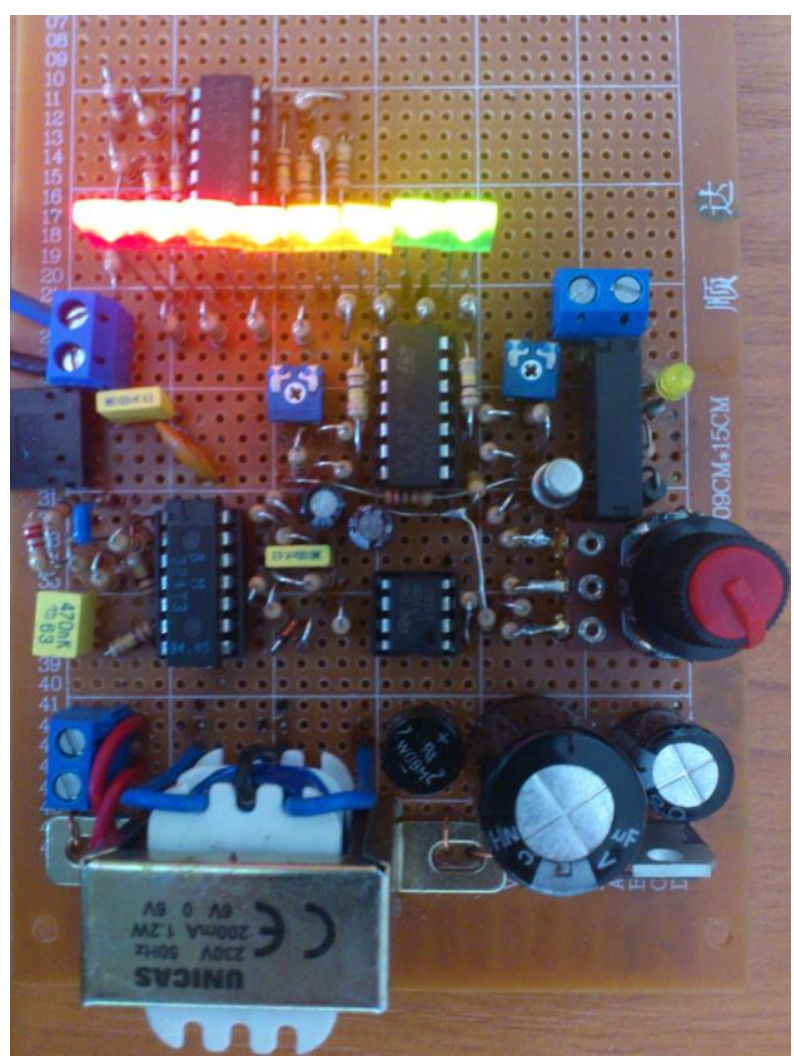

Figure 10. The picture of the prototype.

\section{CONCLUSIONS}

This article presented a device for measuring and maintaining the water level of a tank. First, there were presented three variants of the capacitive sensor used for level measurement. Secondly, it was shown the conditions that should be fulfilled for such a device to meet customer requirements.

Based on these requirements, it was presented an embodiment of such a device. The device uses operational amplifiers powered from a single supply voltage $(+5 \mathrm{~V})$, which makes it safe and practical.

It was presented in detail the capacitive water level sensor designed and simulated in the software from National Instruments, Multisim 12.0.

The sensor uses a square wave generator of a few $\mathrm{kHz}$, according to the characteristics of capacitive sensor. The frequency of generator, the sensor capacity and the gain of output amplifier should be correlated so that the output voltage of the device to be in the range of linearity of the output AO LM324, in the domain of $0.5 \mathrm{~V}$ to $3.5 \mathrm{~V}$.

The output voltage is used to measure and control the water level in the tank, but can be used in the context of more elaborate automation.

The experimental results are accordingly with the simulations.

The realizations of the prototype and measurements with three different variants of the sensor of water levels in the tank were presented.

\section{ACKNOWLEDGEMENTS}

In this way we would like to thank to the University Valahia of Targoviste, the Faculty of Electrical Engineering, Electronics and Information Technology for supplying the necessary laboratory equipment to check the results obtained by the simulation.

\section{REFERENCES}

[1] http://www.brighthubengineering.com

[2] www.electroschematics.com.

[3] http://www.instructables.com

[4] G. Socher, Remote flower watering and monitoring, http://tuxgraphics.org/electronics/200908/eth-flowerwatering.shtml

[5] http://www.vegetronix.com/Products/RelayBoards.phtml

[6] A. Vătăşescu, A. Hartular, M. Bodea, Linear integrated circuits - User's guide - Vol 1, Technical Publishing Practical Series, Bucharest, 1984.

[7] ***, Single-Supply Op Amp Design Techniques Application Report, March 2001, sloa030a.pdf. 
[8] R. Râpeanu, O. Chirică, et.all, Analog Integrated Circuits - Publishing Technical Practical Series, Bucharest, 1983.

[9] I. Craiu, Measuring device and maintaining the water level of a tank, Undergraduate work, Valahia University of Targoviste, Faculty of Electrical Engineering, Electronics and Information Technology, Department of Electronics, Telecommunications and Energy Engineering, 2013. 